\title{
Formación tecnológica de la competencia de traducción y el uso de ejercicios comunicativos para desarrollar destrezas de lectura en textos de diferente género en inglés
}

Technological training of translation competence and the use of communicative exercises to develop reading skills in texts of different genres in English

1 Zoila Victoria Herrera Andrade https://orcid.org/0000-0002-4781-7320

Escuela Superior Politécnica de Chimborazo (ESPOCH), Sede Orellana, Orellana, Ecuador zherrera@espoch.edu.ec

2 Nancy Georgina Rodríguez Arella https://orcid.org/0000-0001-8240-3679 Escuela Superior Politécnica de Chimborazo (ESPOCH), Sede Orellana, Orellana, Ecuador nancy.rodriguez@espoch.edu.ec

$3 \quad$ Sandra Leticia Guijarro Paguay https://orcid.org/0000-0002-0413-4925 Escuela Superior Politécnica de Chimborazo (ESPOCH), Facultad de Mecánica. Riobamba, Ecuador sandra.guijarro@espoch.edu.ec

Artículo de Investigación Científica y Tecnológica Enviado: 10/11/2021 Revisado: $25 / 11 / 2021$

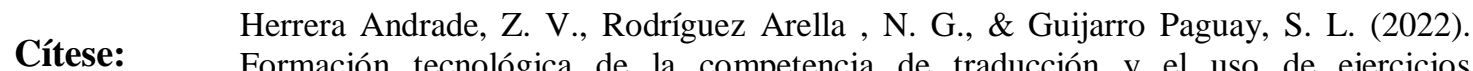
Formación tecnológica de la competencia de traducción y el uso de ejercicios comunicativos para desarrollar destrezas de lectura en textos de diferente género en inglés . ConcienciaDigital, 5(1), 70-87. https://doi.org/10.33262/concienciadigital.v5i1.1965

CONCIENCIA DIGITAL, es una Revista Multidisciplinar, Trimestral, que se publicará en soporte electrónico tiene como misión contribuir a la formación de profesionales competentes con visión humanística y crítica que sean capaces de exponer sus resultados investigativos y científicos en la misma medida que se promueva mediante su intervención cambios positivos en la sociedad. https://concienciadigital.org La revista es editada por la Editorial Ciencia Digital (Editorial de prestigio registrada en la Cámara Ecuatoriana de Libro con No de Afiliación 663) www.celibro.org.ec 
Palabras claves: comunicación; funciones; habilidades; tipos de lectura; tecnología; semántica del inglés

\section{Keywords:}

communication; functions; skills; types of reading; technology; semantics of English.
Resumen

El artículo está dedicado a la tecnología del uso de ejercicios comunicativos en la enseñanza de la lectura, que ayudan a enseñar a los estudiantes cómo trabajar con textos de diferentes géneros y desarrollar y mejorar esta habilidad. La referencia repetida al texto de acuerdo con esta tecnología desarrolla no solo la capacidad de leer, sino que también le permite alcanzar su comprensión a nivel de significado. Este artículo corresponde a una propuesta considerando una serie de ejercicios comunicativos diseñados para trabajar con textos de diferente tipo y contribuir no solo a la comprensión de lo leído, sino también a la expresión del habla de alta calidad con la expresión de la propia opinión. La experiencia de organizar el trabajo independiente en inglés como lengua extranjera en el nivel 0 - B1, a través las tecnologías informáticas utilizando para ello: parte de las ayudas didácticas electrónicas disponibles en la red de datos. El cambio en el número de horas de carga académica disponible para el aula de clases requiere la actualización y creación de materiales didácticos electrónicos. Por otra parte, la competencia comunicativa de los universitarios en el estudio de una lengua extranjera, empleando métodos innovadores para pronunciar correctamente la lengua inglesa, en esta etapa importante de aprendizaje para la generación de enunciados en forma dialógica como monológica, mediante ejemplos con los que se puede construir una guía didáctica para el manejo de cada tema contemplado en el currículo de inglés.

\section{Abstract}

The article is dedicated to the technology of the use of communicative exercises in the teaching of reading, which help teach students how to work with texts of different genres and develop and improve this skill. Repeated reference to the text according to this technology develops not only the ability to read, but also allows you to achieve your understanding at the level of meaning. This article corresponds to a proposal considering a series of communicative exercises designed to work with texts of different types and contribute not only to the understanding of what is read, but also to the expression of high-quality speech with the expression of one's own opinion. The experience of organizing independent work in English as a foreign language at level 0 - B1, through computer technologies using for it: part of the electronic 
teaching aids available on the data network. The change in the number of hours of academic load available for the classroom requires the updating and creation of electronic teaching materials. On the other hand, the communicative competence of university students in the study of a foreign language, using innovative methods to pronounce the English language correctly, in this important stage of learning for the generation of statements in a dialogical and monological way, through examples with which can build a didactic guide for handling each topic covered in the English.

\section{Introducción}

La formación y existencia de una persona es imposible sin comunicación. La comunicación es un proceso y resultado de establecer contactos entre personas, que tiene muchas funciones. En pocas palabras, estos son los objetivos que persigue una persona a lo largo de su vida en la sociedad y las tareas que resuelve a través de la comunicación (Maura, 2006).

La comunicación tiene una serie de funciones, como:

- $\quad$ coordinación (ayuda a las personas a coordinar acciones conjuntas);

- $\quad$ emotivo (te hace sentir las emociones necesarias para una vida plena);

- $\quad$ función de autoexpresión (es un medio de revelar la propia esencia);

- función de socialización (en el proceso de socialización, una persona aprende a seguir estándares normativos generalmente aceptados) (Llorca, 2016).

Sin, embargo las funciones que realiza la comunicación también se relaciona con las actividades receptivas, es decir, con la lectura y la escucha (Chacón, 2006). En este caso, una persona no actúa sobre su interlocutor, sino sobre sí misma a través de un libro, artículo, material de referencia, esforzándose así por satisfacer sus necesidades:

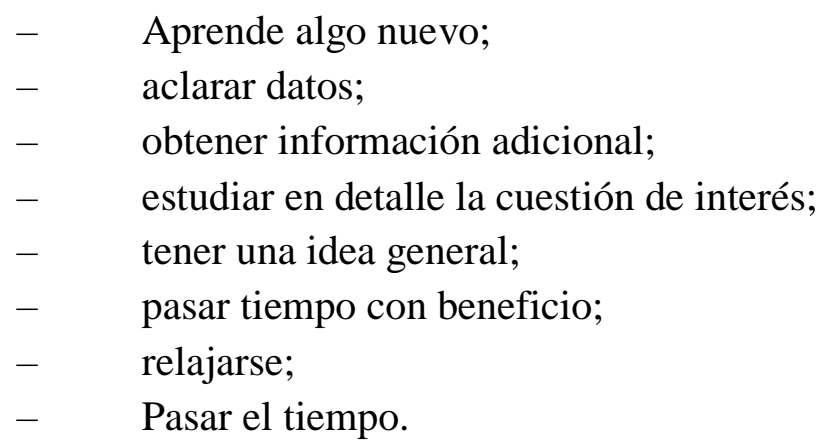


En el proceso de lectura, se forman las siguientes habilidades y destrezas:

1) habilidades de lectura léxica;

2) habilidades de lectura gramatical;

3) habilidades de lectura perceptiva (técnica de lectura);

4) la capacidad de elegir material en función de intereses, deseos y necesidades;

5) capacidad para leer rápidamente;

6) la capacidad de adivinar;

7) la capacidad de anticipar;

8) la capacidad de usar un diccionario y libros de referencia;

9) la capacidad de comprender la idea principal y el significado;

10) la capacidad de comprender el contenido general del texto;

11) la capacidad de elegir lo principal del texto.

Estas habilidades se desarrollan en el proceso de lectura de textos de diferentes géneros (ficción, periodística, científica, referencial, etc.) (Rico-Martín, 2005).

Ser capaz de leer significa no solo dominar la técnica de lectura (reconocer visualmente las unidades del habla), sino también correlacionar rápidamente estas unidades de habla y diseño gramatical con su significado. Esta comprensión se basa en la capacidad de anticipar tanto el significado del texto como las construcciones gramaticales individuales (Fontecha, 2001).

Una suposición semántica juega un papel igualmente importante, es decir, la capacidad de comprender una palabra desconocida en función del contexto.

Una persona que sepa leer no solo debe dominar todos los tipos de lectura, sino que también debe cambiar fácilmente de un tipo a otro, dependiendo del cambio en el propósito de obtener información de este texto (Salinas, 2004).

Según el objetivo, se distinguen los siguientes escenarios: visionado (leer con una comprensión general, sin entrar en detalles), informativo (extraer información básica del texto), cognitivo (la comprensión más completa y precisa de toda la información contenida en el texto y su comprensión) y lectura de búsqueda (encontrar rápidamente los hechos, características, fechas, instrucciones, etc. necesarios en el texto) (Martín, 2004).

La enseñanza del inglés como lengua extranjera (en adelante, EFL) está pasando por momentos difíciles. La educación en inglés está regulada por el estándar educativo del Ecuador.

La implementación del nuevo estándar educativo para la formación profesional incluye el inglés en la etapa universitaria y se han encontrado ciertas dificultades. Estas dificultades están asociadas con las actividades del profesor, y con el nivel de preparación 
de los estudiantes, y con dificultades absolutamente objetivas en la enseñanza de una lengua como medio de comunicación, frente a la enseñanza de otras disciplinas (Mendoza, 2013).

Los estudiantes que planifican visitar un país extranjero pasan objetivamente por un período de adaptación sociocultural; muchos estudiantes no están en absoluto preparados para realizar un trabajo independiente, ya que no han recibido tal habilidad en casa (Boehm, 2015).

La falta de mecanismos efectivos para contabilizar los costos del maestro reduce la motivación del maestro cualitativamente, estas dificultades están asociadas con las actividades del profesor, y con el nivel de preparación de los estudiantes, y con dificultades absolutamente objetivas en la enseñanza de una lengua como medio de comunicación, frente a la enseñanza de otras disciplinas. Los estudiantes pasan objetivamente por un período de adaptación sociocultural; muchos estudiantes no están en absoluto preparados para realizar un trabajo independiente, ya que no han recibido tal habilidad en casa (Cao, 2009).

El término "ES" es una actividad organizada por la propia persona en virtud de sus motivos cognitivos internos y realizada en el momento más conveniente, controlado por él en el proceso y como resultado, realizado sobre la base del manejo sistémico mediado externo del mismo por un profesor o un programa de formación (Escobar, 2010).

Es conveniente mencionar que en las condiciones modernas el aprendizaje del inglés es el trabajo que realiza el alumno siguiendo las instrucciones del maestro, pero sin su participación directa (Puryear, 2007).

Una de las direcciones más importantes en la enseñanza de un idioma extranjero a estudiantes universitarios es la formación de habilidades de comunicación dialógica de orientación profesional. Este último se refiere al tipo de comunicación que puede surgir a través del contacto directo con profesionales de otros países. La organización de relaciones de asociación y la interacción estratégica con otros países a través del establecimiento de un diálogo es de primordial importancia. La construcción de relaciones y lazos entre países aliados y adversarios requiere preparación y la capacidad de llevar a cabo una comunicación profesional con representantes de otras culturas (Díaz, 2016).

En la actualidad los egresados universitarios pueden enfrentar la situación real de comunicarse con extranjeros. El objetivo de un profesor de lengua extranjera es formar profesionales con altas capacidades de comunicación (Pulido, 2005).

Dado que el objetivo principal de la metodología comunicativa es la enseñanza de una lengua extranjera como medio de comunicación, en el aula debe haber capacitación en la 
generación de enunciados en lenguas extranjeras tanto en forma dialógica como monológica. Tanto el discurso dialógico como el monólogo tienen la función de comunicación, intercambio de pensamientos y sentimientos entre las personas (Bosa, 2010).

Sin embargo, el discurso dialógico está destinado al intercambio directo de información entre dos o más participantes en la comunicación. Está diseñado para resolver problemas tales como el desarrollo de la capacidad de hacer una pregunta, responderla, comunicar información, expresar una actitud personal ante los mensajes generados (Téllez, 2017).

El discurso monólogo es, en su mayor parte, un vector por naturaleza. Realiza otras funciones: informativa, influyente, evaluativa. El diálogo es un medio bastante eficaz de desarrollar la competencia comunicativa de lengua extranjera debido a las amplias posibilidades en combinación con un gasto mínimo de tiempo y resultados efectivos (Rodríguez, 2014).

En las clases prácticas, se utilizan los siguientes tipos de actividades para ayudar a formar y desarrollar la capacidad de hablar: juego de roles, discusión, descripción de fenómenos, esquemas, objetos, traducción y varios ejercicios.

\section{Metodología}

El material seleccionado para el desarrollo de este tipo de lecturas es completamente diferente. Debe corresponder a los intereses y necesidades comunicativo - cognitivas de los estudiantes, contener información interesante y adecuada en cuanto al grado de complejidad para cada grupo de edad. En consecuencia, el método de trabajar con estos textos también debería ser diferente.

En su práctica, el maestro, por regla general, combina tecnologías y métodos tradicionales e innovadores, según los objetivos, las condiciones, el aprendizaje y otros factores.

En este artículo se muestran algunas lecciones de lectura, dando preferencia a los ejercicios de comunicación que ayudan no solo a enseñar a los estudiantes a trabajar con textos de diferentes tipos, sino también a desarrollar y mejorar esta habilidad.

Por ejemplo, una lección que utiliza el componente social "Grandes personalidades de Ecuador y Latinoamérica ". La lección comienza con una exposición en la que el docente inicia una conversación sobre lo que significa el concepto de Personalidades y representantes sociales, brinda a los estudiantes los conocimientos básicos necesarios para el trabajo posterior con los textos y los anima a formular el tema de la lección por su cuenta.

Ejemplo: exposición: Cada país tiene su propia gente sobresaliente que merecen ser admirados y respetados por sus logros y hazañas heroicas. 
- ¿ ¿De qué gente está orgulloso nuestro país?

- ¿Por qué son famosos?

- ¿QQué personas destacadas de Ecuador puedes nombrar?

- ¿ ¿Qué hicieron por su país?

- $\quad$ Hay un lema popular en nuestra sociedad llamado "Unidad en la diversidad". Se basa en la comprensión que enriquece nuestras interacciones humanas y nos ayuda a comprendernos mejor.

Inmediatamente después de la exposición, hay una pregunta principal que ayuda a los estudiantes a concretar el tema de la lección y desarrollar la capacidad de predecir.

Por ejemplo:

1. Lea los textos breves sobre los grandes personajes de Ecuador y Latinoamérica y diga si hay alguno que ya haya mencionado. Sugiera su propia variante del tema de nuestra lección.

a) Abdón Calderón fue un militar al servicio del ejército Libertario para la Independencia del Ecuador. Luchó en la batalla de Pichincha y fue declarado héroe niño.

b) Antonio José Francisco de Sucre y Alcalá (Cumaná, 3 de febrero de 1795 Berruecos, 4 de junio de 1830), también conocido como Gran Mariscal de Ayacucho, fue un político, mariscal y militar venezolano, prócer de la emancipación hispanoamericana y principal héroe de la actual República del Ecuador, así como un diplomático y estadista, presidente de Bolivia, gobernador del Perú, General en jefe del Ejército de la Gran Colombia, comandante del Ejército del Sur y Gran Mariscal de Ayacucho.12345.

c) Simón José Antonio de la Santísima Trinidad Bolívar Palacios Ponte y Blanco (Caracas, 24 de julio de 1783 -Santa Marta, 17 de diciembre de 1830), más conocido como Simón Bolívar, fue un militar y político venezolano, fundador de las repúblicas de la Gran Colombia y Bolivia. Fue una de las figuras más destacadas de la emancipación hispanoamericana frente al Imperio español. Contribuyó a inspirar y concretar de manera decisiva la independencia de Bolivia, Colombia, Ecuador, Panamá, Perú y Venezuela.

2. José Eloy Alfaro Delgado fue presidente de la República de Ecuador en dos ocasiones en períodos que comprenden entre 1895 a 1901 y 1906 a 1911, general de división del Ejército del Ecuador desde 1895 y líder de la revolución liberal ecuatoriana.

3. ¿Quiénes son las personas descritas en estos textos? 
a) Científicos famosos

b) Personajes de ficción

c) Escritores famosos

d) Líderes políticos y militares

A continuación, el trabajo comienza con textos utilizando ejercicios de comunicación directa. Cada ejercicio comienza con una actitud comunicativa, que debe entenderse como una declaración escrita u oral del docente, que sirve como una forma de controlar la actividad del habla de los estudiantes en una situación determinada y de acuerdo con la tarea comunicativa recibida. La actitud comunicativa estimula y regula los procesos de pensamiento, emocionales, intelectuales y otros asociados con la actividad educativa y cognitiva de los estudiantes.

Por ejemplo:

Lea los textos una vez más para encontrar los hechos que prueban que existen similitudes entre estas personas.

Luego se realizan tres grupos de ejercicios. El primer grupo se llama identificación de contenido. Estos ejercicios sirven para que el alumno pueda correlacionar el contenido de la información leída con las frases ofrecidas por el profesor que tienen un significado similar. Son posibles las siguientes variantes de tales ejercicios: encontrar oraciones en el texto leído que sean similares en contenido a los datos; definir, cumplimiento de estas frases con el texto; encontrar en el texto frases que amplíen los enunciados dados, etc.

Por ejemplo, uno de los ejercicios de este tipo se puede representar de la siguiente manera:

En los textos breves sobre hechos, busque frases que sean similares a estas.

a) Se centró en las relaciones con países extranjeros.

b) Se hizo famoso después de organizar la defensa de una ciudad del Ecuador.

c) Su talento militar lo ayudó a ganar en muchas batallas.

d) Trató de cambiar algunas leyes agrarias.

e) A pesar de estar herido, logró llegar al territorio no controlado por el enemigo.

El segundo grupo de ejercicios se llama búsqueda de contenido. Estos ejercicios tienen como objetivo desarrollar el mecanismo de comprensión lógica y recuperación de información. Las tareas de este tipo se pueden formular de la siguiente manera: encontrar las razones por las que ...; encontrar propuestas que confirmen ......; encontrar respuestas en el texto .... etc.

Un ejercicio de ejemplo para este grupo podría ser el siguiente:

Demuestre con el texto que ....... 
a) Abdón Calderón fue un verdadero patriota del ejército libertario.

b) Eloy Alfaro fue un talentoso líder militar.

c) Simón Bolívar fue un importante líder político de la Gran Colombia.

d) Antonio José de Sucre mostró un ejemplo de increíble coraje durante las guerras de la Independencia.

El tercer grupo de ejercicios se llama elección semántica. La tarea de estos ejercicios es desarrollar no solo la comprensión lógica, sino también las conjeturas semánticas. Al realizar tales ejercicios, el estudiante debe elegir no solo la respuesta correcta, sino también poder confirmarla.

Los siguientes son posibles variantes de tales ejercicios: restaurar la información semántica; escriba una anotación al texto; elija la respuesta correcta de las sugeridas, etc., por ejemplo:

Restaura la información faltante para hacer un cartel sobre las grandes personalidades de Ecuador y América Latina.

a) Simón Bolívar es uno de los personajes más famosos en la historia de la independencia de las colonias Sudamericanas. Su mejor momento llegó a inicios del siglo XIX

b) Antonio José de Sucre era famoso por su liderazgo inspirador, excelente dominio de la estrategia y obtuvo en una serie de victorias decisivas. Fue herido de manera significativa varias veces en combate.

No menos importante es la etapa final del trabajo con textos, cuyo propósito es estimular a los estudiantes a hablar en forma oral con la expresión de su propia opinión y actitud ante lo que leen. Ejemplos de tareas que inducen el habla pueden ser:

1. Busque parejas de personas que tengan algo en común y complete el cuadro con sus imágenes.

2. Elija un par de personas que le hayan impresionado más y proporcione un breve informe sobre las similitudes en sus vidas y carreras. Utilice el modelo de voz si lo necesita.

Me gustaría contarles sobre ...... y ...... Tienen mucho en común.

Primero ...... Segundo... Finalmente...

Creo que merecen ser llamados las grandes personalidades de sus países.

La tecnología del uso de ejercicios de comunicación es universal para textos de varios géneros. Se sabe que a muchos adolescentes les encanta leer aventuras y cuentos. Leerlos genera muchas preguntas, emociones y, lo más importante, el deseo de seguir leyendo. 
Por eso, como otro ejemplo, nos gustaría ofrecer opciones de trabajos con textos del género de ficción, que se encuentran en el kit didáctico y metodológico de la serie "English in Focus" para los grados 10-11 de educación general.

A continuación, los elementos de una lección basada en un extracto de la novela El mundo perdido de Arthur Conan Doyle.

I. Exposición (en forma de conversación)

Mucha gente disfruta leyendo historias de aventuras y artículos sobre viajes.

- $\quad$ ¿Disfrutas leyendo esas historias? ¿Por qué? ¿Los lee a menudo?

- ¿Quién es tu escritor de historias de aventuras favorito?

- $\quad$ ¿Te gusta ver películas de aventuras? ¿Les parecen emocionantes? ¿Por qué?

- $\quad$ (Mostramos un pequeño fragmento de la película "El mundo perdido" (2001)

- ¿ ¿Sabes en qué libro se basó la película?

- $\quad$ ¿Quién escribió este libro? Pregunta principal.

Lea la breve biografía del autor y averigüe si tenía razón.

II. Identificación de contenido.

Busque en el texto oraciones que amplíen y detallen la información que se proporciona a continuación.

a) El profesor Summerlee estaba feliz con su hallazgo.

b) Había visto esas huellas antes.

c) Las pistas los llevan a algo extraordinario.

d) Las criaturas parecían grandes reptiles.

e) Las criaturas estaban ocupadas con algo.

f) Las criaturas no pudieron notar a los viajeros.

g) Las criaturas eran fuertes, pero no muy inteligentes.

h) Los viajeros estaban demasiado asombrados para hablar.

i) Los viajeros no estaban seguros de que los demás les creyeran.

III. Búsqueda de contenido.

Encuentra en el texto frases que caracterizan ...

a) la reacción de los viajeros tras el primer hallazgo.

b) las asombrosas criaturas

c) el comportamiento de las criaturas

d) el asombro y las dudas de los viajeros.

e) Elección semántica. 
f) Elija el encabezado alternativo más apropiado para el extracto.

g) Un hallazgo extraordinario.

h) Criaturas parecidas a reptiles.

i) Increíble expedición.

2. Encuentre oraciones o frases clave en cada párrafo del extracto.

IV. La etapa final de trabajo con el texto. (motivación de los estudiantes para pronunciar el habla)

Los viajeros dudan que alguien pueda creer en lo que han visto en la selva amazónica de América del Sur. ¿Cómo intentarías persuadir a los escépticos si participaras en esa expedición? Siga el modelo de voz si lo necesita.

¡Nuestra expedición a Sudamérica fue increíble!

Atravesando la selva amazónica, de repente nos encontramos con ...

No creerías eso ...

Pero lo que más me impresionó fue ... Entonces, tomé mi diario y escribí allí ...

Cada módulo del conjunto pedagógico-metódico de la serie "An-Focus on Glya" para los grados 10-11 contiene una sección llamada Rincón cultural que contiene textos breves que brindan una idea de la cultura y la vida de los países de habla inglesa. Teniendo en cuenta que viajar es una de nuestras actividades de ocio favoritas, decidimos llevar a cabo una de las lecciones de esta sección utilizando folletos de viajes auténticos en lugar del texto sugerido sobre Madame Tussauds.

\section{Exposición (en forma de conversación).}

A muchas personas les gusta viajar y visitar diferentes museos y galerías.

- ¿ ¿Disfruta visitando museos y galerías? ¿Por qué? ¿Los visita a menudo?

- $\quad$ ¿Cuál es el último museo o galería en el que has estado?

\section{Pregunta principal.}

Eche un vistazo a algunas de las atracciones turísticas británicas en Londres.

Adivina por qué son famosos.

Atracciones turísticas británicas en Londres. Por que son famosos

El ojo de Londres. Allí podrá disfrutar de hermosas obras de arte moderno. 
Museo de Historia Natural. Allí puede aprender sobre los logros tecnológicos.

Museo Madame Tussauds. Puedes echar un vistazo a la ciudad a vista de pájaro.

Museo de Victoria y Alberto. Puedes ver esqueletos de dinosaurios allí.

Torre de Londres. Este lugar tiene una gran colección de arte y diseño.

Museo de Ciencia. Este lugar exhibe las esculturas de cera de personajes históricos y famosos y personajes de películas.

Tate moderno. Allí se pueden admirar las Joyas de la Corona.

\section{Identificación de contenido.}

Lea los anuncios y compruebe si tenía razón (los alumnos leen los anuncios y comprueban si su elección fue correcta).

\section{Búsqueda de contenido.}

Examine las siguientes palabras clave. Adivina qué lugar de interés describe cada uno de ellos...
a) Artes decorativas y diseño
b) Dinosaurios y otros especímenes
c) Joyas valiosas
d) Registros y logros científicos

Arte moderno internacional

e) Modelos de cera

f) Vista impresionante

a) V. Elección semántica.

Imagina que estás en Londres. ¿A dónde podrías ir si ...?
a) estás en la estación de Waterloo?
b) son las 9.30 ?
c) ¿le gusta la ciencia?
d) quieres una foto con una celebridad?
e) ¿quieres ver algo único y valioso?
f) estás caminando por Cromwell Road?
g) ¿quieres ver la colección de 4,5 millones de objetos?

VI. La etapa final del trabajo con el texto (motivación de los estudiantes para pronunciar el habla). 
Haga una lista de las tres atracciones turísticas que le gustaría visitar y explique su elección. Siga el modelo de voz si lo necesita.

Si estuviera en Londres visitaría..., y.... Mi primera prioridad es ... porque ...

Entonces iría a... porque.... Finalmente, me dirigiría a ... porque ...

Estoy seguro de esto haría mi excursión....

Entonces, ¿cuáles son las ventajas de la tecnología de usar ejercicios comunicativos en la enseñanza de la lectura?

En primer lugar, estos ejercicios son universales y bastante efectivos cuando se trabaja con textos de diferentes géneros.

En segundo lugar, implican referencias repetidas al texto, lo que permite a los estudiantes comprender el texto de forma semántica.

Y finalmente En tercer lugar, al realizar estos ejercicios de forma secuencial, el alumno alcanza un nivel suficientemente alto de comprensión lectora, lo que elimina la necesidad de un control abierto.

\section{Resultados}

En este caso resulta relevante el tema del control sobre la enseñanza del inglés realizada. Los metodólogos que se ocupan de este problema señalan que la valoración del método de enseñanza puede incluirse en la valoración final de la disciplina, para ello, por ejemplo, se puede llevar un diario aparte.

Sin embargo, el punto no está solo en la organización formal (aunque también), sino en el hecho de que, de los cuatro tipos de actividad del habla, dos (hablar y escribir) no están controlados por una máquina y requieren un trabajo individual.

En este sentido, la enseñanza del inglés con el uso de tecnologías informáticas, es decir, con un programa informático de enseñanza / seguimiento, puede incluir todo tipo de actividad del habla, pero la etapa de control del habla y la escritura implica la actividad del docente.

Actualmente, el uso de tecnologías informáticas en el proceso educativo, o aprendizaje electrónico (e-learning), ofrece las siguientes opciones para organizar y monitorear la implementación de la enseñanza del inglés:

- $\quad$ autoaprendizaje (trabajo independiente con materiales educativos electrónicos o recursos de Internet); 
aprendizaje guiado (trabajo independiente de los estudiantes en el aula bajo la dirección de un profesor).

Los materiales didácticos electrónicos se pueden crear sobre la base de varios sistemas de software. Los más comunes son LMS (Learning Management Systems), LCMS (Learning Content Management Systems), como Moodle, Blackboard, etc. Estos sistemas son inherentemente sitios de Internet con capacidades limitadas para la presentación de materiales educativos, pero hasta ahora los más accesible a las instituciones educativas. Menos comunes, pero más efectivos en el proceso educativo, son los desarrollos de software de autor. En este caso, los materiales de enseñanza electrónicos son creados por programadores en cooperación con los profesores, metodólogos para la creación de contenido electrónico, lo que significa que las capacidades de enseñanza de las tecnologías informáticas se realizan en mayor medida.

En el formato de estos dos sistemas de software, los programadores del laboratorio, metodólogos y profesores del departamento de lengua inglesa han creado materiales electrónicos educativos y de control que se utilizan con éxito en la enseñanza. EFL, en particular para organizar el trabajo independiente de los estudiantes.

Los estudiantes de la etapa preuniversitaria de la enseñanza de la lengua inglesa tienen la oportunidad de trabajar de forma independiente con los siguientes materiales electrónicos:

- Ayudas didácticas multimedia interactivas sobre gramática, que contienen una presentación multimedia de material educativo, formación interactiva, control en forma de prueba, libro de consulta gramatical;

- pruebas electrónicas de entrenamiento y control en vocabulario, gramática, fonética, comprensión auditiva y lectura. La funcional del "Sistema de Pruebas Educativas" permite automatizar la verificación de las pruebas completadas, corregir las respuestas incorrectas de los estudiantes, acumular estadísticas individuales y grupales sobre la aprobación de las pruebas por parte de los estudiantes durante el año académico;

- lecciones de video interactivas con un video educativo y una conferencia en video por un profesor de gramática, complementada con tareas de prueba interactivas y material de referencia;

- aplicaciones interactivas para publicaciones impresas, incluidos textos sonoros y diálogos, tareas destinadas a monitorear la comprensión del contenido del texto.

Posibles modos de trabajo independiente de los estudiantes con datos:

- en una clase de inglés bajo la dirección de un maestro;

- en una clase de inglés después del horario escolar; 
- de forma remota si el estudiante tiene una computadora portátil con Windows. En condiciones modernas, trabajo independiente a distancia que los estudiantes son considerados como uno de los más modernos

Las posibilidades de reorganizar el proceso de la educación universitaria con énfasis en una mayor independencia y autonomía de los estudiantes. Sin embargo, los estudiantes solo tienen teléfonos inteligentes a su disposición, en el mejor de los casos, tabletas y el trabajo independiente en una computadora es inaccesible para muchos de ellos. En este sentido los docentes de inglés están desarrollando un proyecto científico y metodológico "Aula virtual inglesa", en cuyo marco la búsqueda de las soluciones óptimas en el campo de la enseñanza de idiomas electrónica (incluida a distancia) y la creación de un entorno educativo electrónico.

\section{Conclusiones}

- El Sector de formación virtual contiene actualmente cuatro secciones (películas instructivas, materiales de audio, tutoriales en vídeo. Referencias). La estructura de cada sección es multinivel, lo que permite llenarla con materiales de diversos temas.

- Las secciones se pueden ampliar según los materiales que se necesiten para el acceso general de estudiantes y profesores con recursos como diccionarios temáticos, textos educativos, pautas, etc.

- Los recursos tecnológicos permiten resolver varios problemas de sistematización y acumulación de materiales multimedia y educativos (video, audio, textos, ilustraciones, libros de referencia, diccionarios

- El aprendizaje del inglés proporciona a los profesionales en las diversas ramas de formación materiales abiertos para uso remoto e independiente a través de dispositivos móviles (teléfonos inteligentes, tabletas).

- El desarrollo del entorno informativo y educativo, complementando los medios electrónicos existentes mejoran significativamente el aprendizaje del inglés con materiales educativos para el trabajo independiente (remoto) de los estudiantes fuera de la institución educativa.

\section{Referencias bibliográficas}

Boehm, S. (2015). Actividades dramáticas en la enseñanza de lenguas extranjeras. Un medio apropiado para enseñar aspectos interculturales. Multidisciplinar, 18.

Bosa Higarrero, L. A. (2010). Criterios para el diseño de fichas didácticas que promueven el desarrollo de la competencia comunicativa en la lengua extranjera-inglés mediante el uso de canciones infantiles norteamericanas. Bachelor. 
Cao, E. R. (2009). El proceso de dirección del trabajo independiente: una vía para la autonomía de los estudiantes. Cuadernos de educación y desarrollo,

Chacón, C. (2006). Formación inicial y competencia comunicativa: percepciones de un grupo de docentes de inglés. Educere, 10(32), 121-130.

Díaz Hidalgo, L. M. (2016). Recursos digitales como estrategia didáctica para el mejoramiento de la competencia comunicativa del inglés en docentes de primaria en la institución educativa Manuel José Sierra del municipio de Girardota. Maestros de primaria.

Escobar, Y. C. (2010). Interdisciplinariedad: desafío para la educación superior y la investigación. Revista Luna Azul, (31), 156-169.

Fontecha, A. F. (2001). Una selección bibliográfica sobre el método AICLE (Aprendizaje Integrado de Conocimientos curriculares y Lengua Extranjera). Contextos Educativos. Revista de Educación, (4), 217-239.

Llorca, C. M. (2016). Hacia una dimensión crítica en la enseñanza de español como lengua extranjera: La Competencia Comunicativa Intercultural Crítica (CCIC). Revista Española de Lingüística Aplicada/Spanish Journal of Applied Linguistics, 29(1), 191-211.

Martín Peris, E. (2004). Las actividades de aprendizaje en los manuales de español como lengua extranjera. Biblioteca virtual redELE.

Maura, V. G. (2006). La formación de competencias profesionales en la universidad: reflexiones y experiencias desde una perspectiva educativa. En-clave pedagógica, 8.

Mendoza Fillola, A. (2013). Literatura, cultura, intercultural. Reflexiones didácticas para la enseñanza de español lengua extranjera.

Pulido Díaz, A. (2005). Propuesta de estrategia didáctica desarrolladora, para concebir el proceso de enseñanza-aprendizaje de la competencia comunicativa integral de la lengua inglesa, en alumnos de 6to grado de la escuela primaria en Pinar del Río.

Puryear, J. (2007). La educación en América Latina: Problemas y desafíos. Santiago: Preal., (Vol. 7, pp. 4-7).

Rico-Martín, A. M. (2005). De la competencia intercultural en la adquisición de una segunda lengua o lengua extranjera: conceptos, metodología y revisión de métodos. 


\section{Conciencia}

Rodríguez Peña, J. C. (2014). La competencia comunicativa oral profesional pedagógica en inglés de los estudiantes de la carrera licenciatura en educación especialidad lenguas extranjeras inglés.

Salinas, J. (2004). Innovación docente y uso de las TIC en la enseñanza universitaria. RUSC. Universities \& Knowledge Society.

Téllez Martínez, I. K. (2017). El Blog Interactivo como Recurso Didáctico para el Desarrollo de la Competencia Comunicativa en Estudiantes de Nivel Medio Superior.

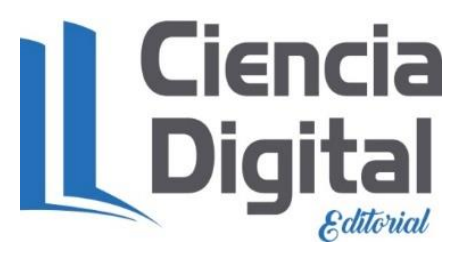




\section{Conciencia}

El artículo que se publica es de exclusiva responsabilidad de los autores y no necesariamente reflejan el pensamiento de la Revista Conciencia Digital.

\section{\Ciencia}

El artículo queda en propiedad de la revista y, por tanto, su publicación parcial y/o total en otro medio tiene que ser autorizado por el director de la Revista Conciencia Digital.
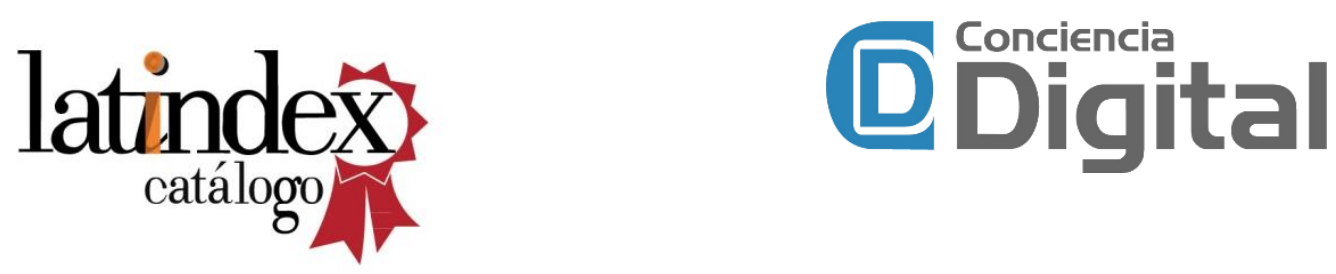

Indexaciones

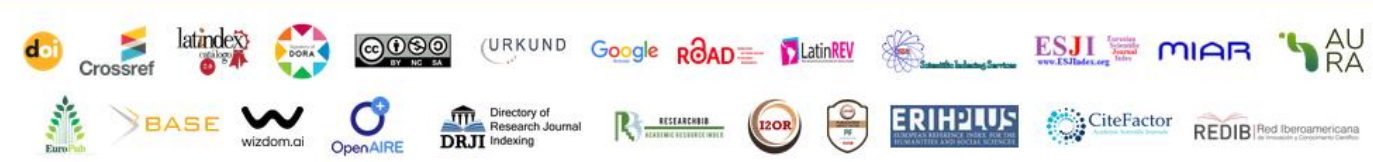

